\title{
Enhancing dynamic actuation performance of dielectric elastomer actuators by tuning viscoelastic effects with polar crosslinking
}

\author{
Matthew Wei Ming Tan', Gurunathan Thangavel ${ }^{1}$ and Pooi See Lee (i)
}

\begin{abstract}
Dielectric elastomer actuators (DEAs) have shown great potential in the field of robotics, energy harvesting, or haptics for wearables. However, existing DEA materials typically require prestretching and exhibit time-dependent deformations due to their inherent viscoelastic properties. In this work, we address these issues by designing and synthesizing a polyurethane acrylate (PUA) DEA copolymerized with a polar crosslinker, polyethylene glycol diacrylate (PEGDA), to reduce viscoelastic effects through chemical crosslinking. We realized a buckling-mode actuator that displays out-of-plane deformations triggered by an electric field without the need for prestretching. Copolymerization with PEGDA showed improved dynamic response actuation performances compared to pristine PUA, wherein the former reached $90 \%$ of its maximum actuation in $<1 \mathrm{~s}$. In addition, precise and stable actuation was achieved, reducing viscoelastic drifts to a negligible amount. Despite the higher elastic modulus of the DEA incurred by the chemical crosslinks, the polar groups present in the PEGDA comonomer effectively increased the dielectric constant. As such, a higher area strain was achieved in comparison to that exhibited by low viscoelastic elastomers such as silicone. By eliminating the need for prestretching, rigid components can be avoided, thereby enabling greater prospects for the integration of fast response and stable DEAs into soft bodies.
\end{abstract}

\section{Introduction}

Dielectric elastomers (DEs) are a class of smart materials that have the ability to convert mechanical energy into electrical energy and vice versa ${ }^{1,2}$. The basic structure of a dielectric elastomer actuator (DEA) device is composed of a DE film sandwiched between two compliant conductive electrodes. When a DEA is subjected to a voltage, opposite charges accumulate at the two electrodes, leading to compression of the DE film due to the Maxwell pressure $P$. As a result of this compressive force, the thickness of the DE film is reduced while the area expands. The Maxwell pressure $P$ generated is expressed in Eq. 1 , where $\varepsilon_{0}$ is the vacuum permittivity, $\varepsilon$ is the dielectric constant, $V$ is the voltage applied across the dielectric and $z$ is the thickness of the dielectric material. At strains $<20 \%$, the DE response is often assumed to be

\footnotetext{
Correspondence: Pooi See Lee (pslee@ntu.edu.sg)

${ }^{1}$ School of Materials Science and Engineering, Nanyang Technological

University, 50 Nanyang Avenue, Singapore 639798, Singapore
}

linear, and the actuation strain incurred by the DE in the thickness direction is estimated through Eq. 2, where $Y$ is the elastic modulus and $S_{\mathrm{z}}$ is the strain in the thickness direction.

$$
\begin{aligned}
& P=\varepsilon_{0} \varepsilon\left(\frac{V}{z}\right)^{2} \\
& S_{Z}=\frac{1}{Y} \varepsilon_{0} \varepsilon\left(\frac{V}{z}\right)^{2}
\end{aligned}
$$

Currently, commercially available acrylate-based (e.g., $\mathrm{VHB}^{\mathrm{TM}}$ ) polymers are often used for DEAs because they exhibit large area strains up to $380 \%$ and possess relatively high dielectric constants $(\approx 4.7)$ and breakdown field strengths ${ }^{3-6}$. However, commercial VHB polymers exhibit slow response and recovery times of several hundred seconds due to their highly viscoelastic nature ${ }^{7,8}$. In addition, as a dissipative process, viscoelastic hysteresis has been shown to exert an adverse effect on performance and cause positioning inaccuracy ${ }^{9,10}$. To circumvent these 
issues, materials with low viscoelasticity, such as silicone rubber, are often used to replace VHB polymers. However, these nonpolar polymers exhibit a low dielectric constant $(\approx 2.8)$, which is a critical parameter to achieve high strains in DEAs, as observed from Eq. $1^{6}$. Hence, a large number of studies have focused on increasing the dielectric constant for improved actuation performance of DEAs, thereby achieving a higher area strain and work density. The general approaches that have often been investigated include the addition of ceramic particles with high dielectric permittivity ${ }^{11,12}$, the addition of conductive nanofillers ${ }^{13,14}$ and chemical modification to introduce polar groups ${ }^{15,16}$. Alternatively, DEAs have been prestretched to reduce the effects of viscoelasticity. Some studies have shown that prestretching provides benefits such as a shorter relaxation time, reduced drifting effects and higher electromechanical strain ${ }^{4,17,18}$. However, the prestretched state is often achieved by mounting the $\mathrm{DE}$ film on a rigid frame, which increases the weight of the actuator and limits the incorporation of DEAs into soft and flexible bodies. Additionally, prestretching reduces the lifetime of the device due to stress relaxation and fatigue.

In this work, we designed and prepared a copolymerized polyurethane acrylate (PUA)-based DEA that avoids prestretching and realizes fast response and stable recovery. This goal is achieved by introducing chemical crosslinks to tune the viscoelasticity caused by the sliding of polymer chains across each other. Although the presence of these chemical crosslinks increases the elastic modulus of the $\mathrm{DE}$, the introduction of polar groups that are present in the comonomer enhances the dielectric constant of the $\mathrm{DE}$, counteracting its impact on actuation performances such as the maximum area strain and work density. We present a buckling-mode DEA, which exhibits out-ofplane deflection without prestretching, based on a copolymer with tunable properties provided by adjusting the crosslinking density. At higher crosslink densities, minimal actuation drifts upon cycling can be realized, and response times of $<1 \mathrm{~s}$ have been achieved, which is a substantial feat for DEAs. The rapid response and stable cycling of DEAs make them attractive for integration into soft bodies or wearable textiles, expanding the capabilities of DEAs for tactile sensing, haptic feedback, and soft robotics.

\section{Materials and methods Materials}

CN9021, an aliphatic urethane acrylate oligomer developed for laminating and pressure sensitive adhesives, was purchased from Sartomer Company and was used as received. Polyethylene glycol diacrylate (PEGDA) with a molecular weight of 575 and azobisisobutyronitrile (AIBN) was obtained from Sigma Aldrich and was used as received. Carbon grease was purchased from Nanjing Xilite Adhesive Co. Ltd and was used as received.

\section{Synthesis of PUA-PEGDA copolymer films}

CN9021 was mixed with different concentrations of PEGDA (5, 10, and $15 \mathrm{wt} \%)$, and $1 \mathrm{wt} \%$ of AIBN was added to the mixture and mixed thoroughly. The resultant precursor solution was then cured on a hotplate in an inert environment at $90^{\circ} \mathrm{C}$ for $1 \mathrm{~h}$. Polymer films were carefully peeled out, and an average film thickness of $0.43 \mathrm{~mm}$ was obtained. Pristine CN9021 films were denoted as PUA, whereas copolymer films were denoted as PUA-PEGDA-X, with $\mathrm{X}$ representing the weight percent of PEGDA.

\section{Characterization of chemical structure}

Fourier transform infrared (FTIR-ATR, Perkin Elmer, Frontier) spectroscopy was used to measure the FTIR spectra by scanning each film 32 times with a resolution of $4 \mathrm{~cm}^{-1}$.

\section{Characterization of dielectric properties}

Dielectric constant measurements were performed in the frequency range of $1 \mathrm{kHz}$ to $1 \mathrm{MHz}$ using an Agilent E4980a LCR meter. An AC signal of $1 \mathrm{~V}$ was applied to the samples, and the dielectric constant was calculated from the capacitance measured with the following relationship: $C=\left(\varepsilon_{0} \varepsilon A\right) / d$, where $A$ is defined as the overlapping area between the top and bottom electrodes, $\varepsilon_{0}$ is the vacuum permittivity, $C$ is the measured capacitance, and $d$ is the polymer film thickness. Elastomer samples were placed between two electrodes for measurements.

\section{Characterization of mechanical properties}

Tensile test specimens were prepared in accordance with ASTM D638-14, Type V. Tensile tests were performed on an Instron 5567 universal testing machine at a strain rate of $3.33 \mathrm{~mm} \mathrm{~s}^{-1}$. Loading and unloading cyclic tests were performed at a strain rate of $1.0 \mathrm{~mm} \mathrm{~s}^{-1}$ for 30 cycles with a limiting strain of $100 \%$.

\section{Actuator fabrication}

Polymer films with an average thickness of $0.43 \mathrm{~mm}$ were placed on an in-house glass holder prepared with a $2 \mathrm{~cm} \times 2 \mathrm{~cm}$ hole to allow out-of-plane actuation. Carbon grease was used as the electrode, which was coated over a circular area with a diameter of $3 \mathrm{~mm}$ on the top and bottom surface of the film. Copper tape was used to provide electrical connection to a Unicorn UHP-75 high voltage DC power supply.

\section{Characterization of actuation performance}

The vertical deflection from the center of the electrode was determined with an Epsilon optoNCDT laser 
displacement sensor. For static actuation experiments, the voltage was increased by $0.5 \mathrm{kV}$ every $10 \mathrm{~s}$. Actuation strains were determined by calculating the change in the electrode area through geometric relations. Cyclic actuation measurements were performed using a Trek $^{\mathrm{TM}}$ Model PD05034 high voltage AC power amplifier to amplify the signal generated from the pulse generator. The cyclic measurements were performed at a frequency of $0.5 \mathrm{~Hz}$ with an electric field strength of $10 \mathrm{~V} \mu \mathrm{m}^{-1}$. To evaluate the response times, the generated wave profiles were obtained at a frequency of $0.1 \mathrm{~Hz}$ with an electric field strength of $10 \mathrm{~V} \mu \mathrm{m}^{-1}$. The response time $t_{0.9}$ of the actuator was quantified by the time taken to reach $90 \%$ of the maximum displacement for each cycle. Three samples were tested for each copolymer formulation.

\section{Results and discussion}

\section{PUA-PEGDA structure}

PUA, consisting of a flexible polyether diol segment and a relatively flexible di-isocyanate segment, was copolymerized with PEGDA (Fig. 1a-c) of low molecular weight $(\mathrm{Mw}=575)$. To verify the successful copolymerization between the two compounds, FTIR (Fig. 1e) and NMR (Figs. S1-3, Supplementary Information) measurements were performed. FTIR measurements of the pristine PUA displayed absorption peaks for $\mathrm{CH}_{2}$ at $2940 \mathrm{~cm}^{-1}(-\mathrm{CH}$ stretching consisting of asymmetric $-\mathrm{CH}_{3}$ stretching at $2955 \mathrm{~cm}^{-1}$, asymmetric $-\mathrm{CH}_{2}$ stretching at $2924 \mathrm{~cm}^{-1}$, symmetric $-\mathrm{CH}_{3}$ stretching at $2873 \mathrm{~cm}^{-1}$ and symmetric $-\mathrm{CH}_{2}$ stretching at $\left.2857 \mathrm{~cm}^{-1}\right), \mathrm{H}-\mathrm{C}-\mathrm{H}$ scissor bending at $1457 \mathrm{~cm}^{-1}$ and $=\mathrm{CH}$ stretching at $810 \mathrm{~cm}^{-1}$. For (a)

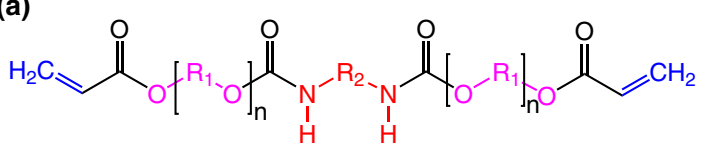

PUA (b)<smiles>C=CC(=O)OCC(C)OC(=O)C=C</smiles>

(c)

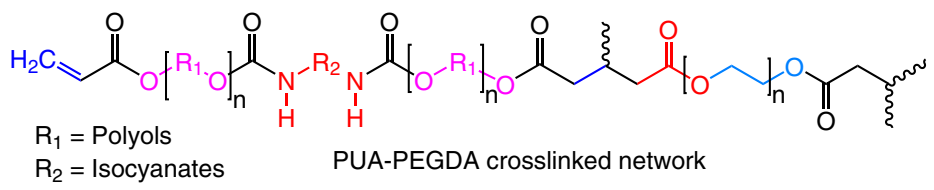

(d)

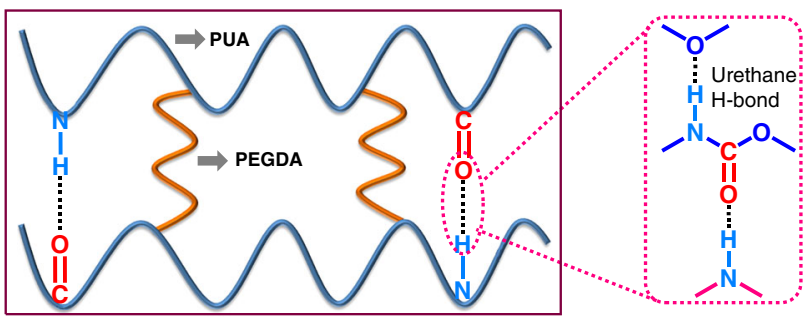

(e)

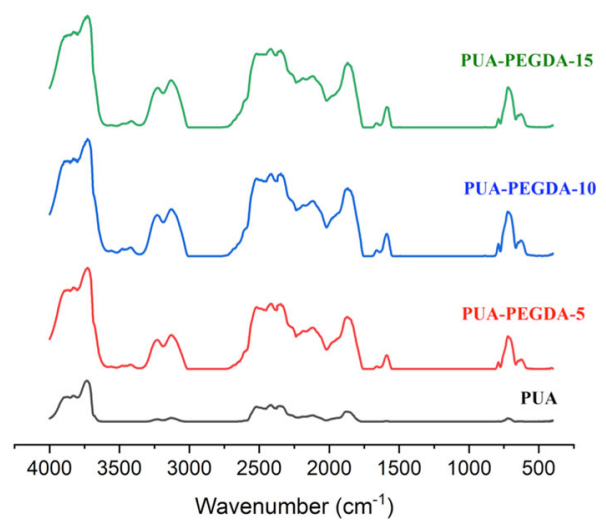

Fig. 1 Characterization of PUA-PEGDA copolymers. Chemical structures of a PUA, b PEGDA, and c PUA-PEGDA copolymer. $\mathbf{d}$ Schematic illustration of crosslinking network structures with PUA-PEGDA. e FTIR spectra of PUA and PUA-PEGDA copolymer films (5, 10, and 15 wt\%) measured over the range of $400-4000 \mathrm{~cm}^{-1}$ 
PUA-PEGDA copolymers, the appearance of strong characteristic peaks (Table S1, Supplementary Information), such as the stretching vibrations of $-\mathrm{NH}-$ at $3350-3200 \mathrm{~cm}^{-1}, \mathrm{C}=\mathrm{O}$ at $1720-1727 \mathrm{~cm}^{-1}, \mathrm{C}=\mathrm{C}$ at 1635 $\mathrm{cm}^{-1}$ and $-\mathrm{NH}-$ at $1520-1580 \mathrm{~cm}^{-1}$, confirms the formation of crosslinks between PEGDA and PUA through free radical polymerization. Furthermore, the spectra do not show the characteristic peak of $-\mathrm{NCO}$ at $2260-2270 \mathrm{~cm}^{-1}$, indicating a complete usage of the isocyanate upon copolymerization. With the successful copolymerization of the two components, a chemical crosslink network between polymer chains is established, preventing the chains from slipping across one another (Fig. 1d).

\section{Dielectric properties of the PUA-PEGDA copolymer}

The dielectric permittivity of the copolymer films with various concentrations of PEGDA is shown in Fig. 2a. With an increase in PEGDA concentration, a drastic improvement in the permittivity is observed. The dielectric permittivity increases from 5.52 to 8.87 at $1 \mathrm{kHz}$ (Fig. 2b), whereas the commercial VHB 4905 (for comparison) has a measured permittivity of 4.4 at $1 \mathrm{kHz}$. This improvement can be attributed to the increase in polarity of the copolymer film due to the increasing number of $\mathrm{C}-\mathrm{O}$ bonds introduced into the polymer with increasing PEGDA content. This finding indicates that the dielectric properties of the films can be tuned by controlling the concentration of PEGDA. This realization is critical because the dielectric permittivity is proportional to the generated Maxwell pressure. As the frequency increases, the permittivity of the copolymer decreases, as illustrated in Fig. 2a. This phenomenon is typically related to dielectric relaxation, in which the polar groups on the polymer chains are unable to keep up with the alternating current, resulting in a loss of contribution to the dielectric permittivity $^{14,15,19}$.

\section{Mechanical properties of the PUA-PEGDA copolymer}

The effects of chemical crosslinks between PEGDA and pristine PUA on the mechanical properties can be observed from the stress-strain curves in Fig. 3a. Copolymer films with concentrations up to $10 \mathrm{wt} \%$ of PEGDA exhibited a nonlinear behavior with a slow, gradual increase in stress at low strains due to the unfolding of polymer chains. However, at larger strains, the elastomer exhibits a strain hardening behavior with a rapid increase in the elastic modulus as the polymer chains begin to become taut and aligned. This elastomeric behavior is a characteristic of many other elastomers, such as polyurethane and VHB polymers ${ }^{20,21}$. At $15 \mathrm{wt} \%$ of PEGDA, the nonlinear elastomeric behavior was attenuated accompanied by a reduced maximum attainable elongation due to an increase in crosslinking. This phenomenon was accompanied by a reduction in the apparent modulus, which may be attributed to an increase in length between crosslink junctions as the probability of PEGDA acrylate groups reacting with each other increases at higher concentrations. As the number of chemical crosslinks between PEGDA and PUA polymer chains increases, the mobility of the chains is severely restricted, reducing the maximum elongation to $167 \%$ from the $960 \%$ elongation of the pristine PUA. Note that the maximum elongation at $15 \mathrm{wt} \%$ of PEGDA is comparable to the maximum elongation of polydimethylsiloxane (PDMS) (Sylgard 184) by Dow Corning ${ }^{22,23}$. A summary of the mechanical properties of the fabricated films is provided in Table 1.

To evaluate the viscoelastic behavior of the fabricated copolymers, loading-unloading cyclic tests were
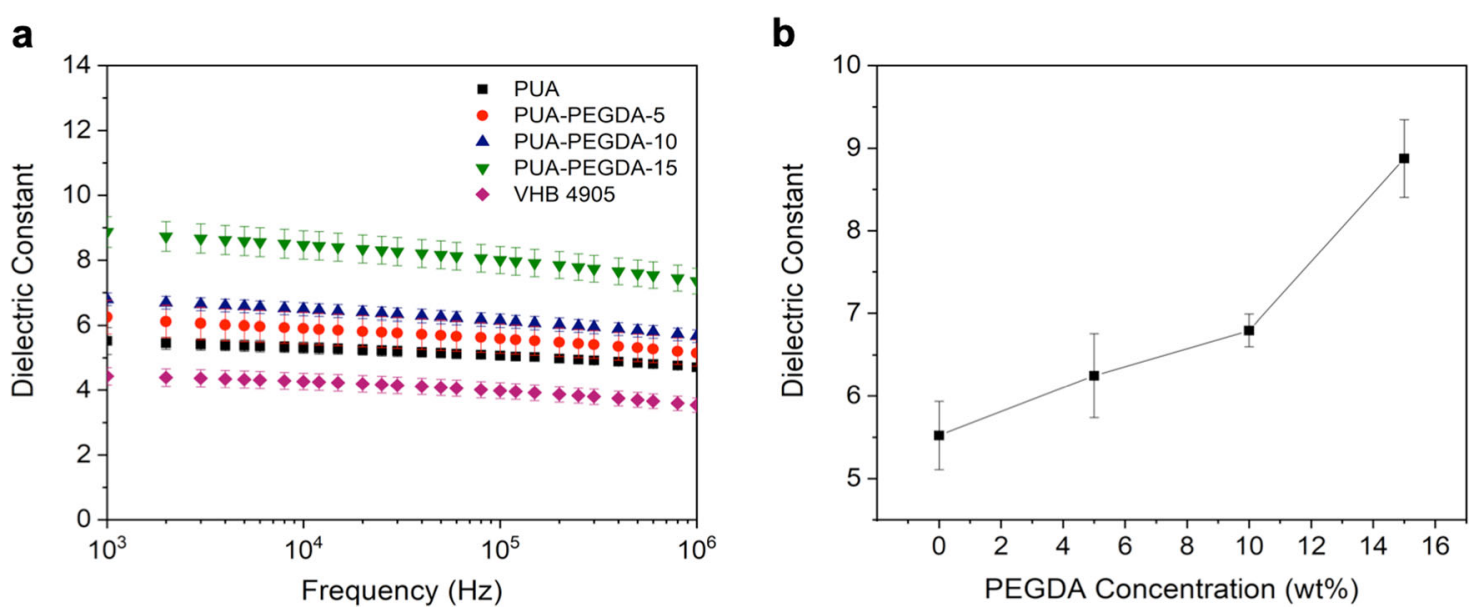

Fig. 2 Characterization of dielectric properties of the PUA-PEGDA copolymers. a Dielectric constants of PUA, PUA-PEGDA copolymer films (5, 10, and $15 \mathrm{wt} \%$ ) and VHB 4905 measured over a frequency range of $1 \mathrm{kHz}$ to $1 \mathrm{MHz}$. b Dielectric constants of copolymer films at various PEGDA concentrations measured at $1 \mathrm{kHz}$. Three samples were measured for each concentration 
performed for 30 cycles at a strain rate of $1 \mathrm{~mm} \mathrm{~s}^{-1}$. From the experiments (Fig. 3b-e), all samples exhibited

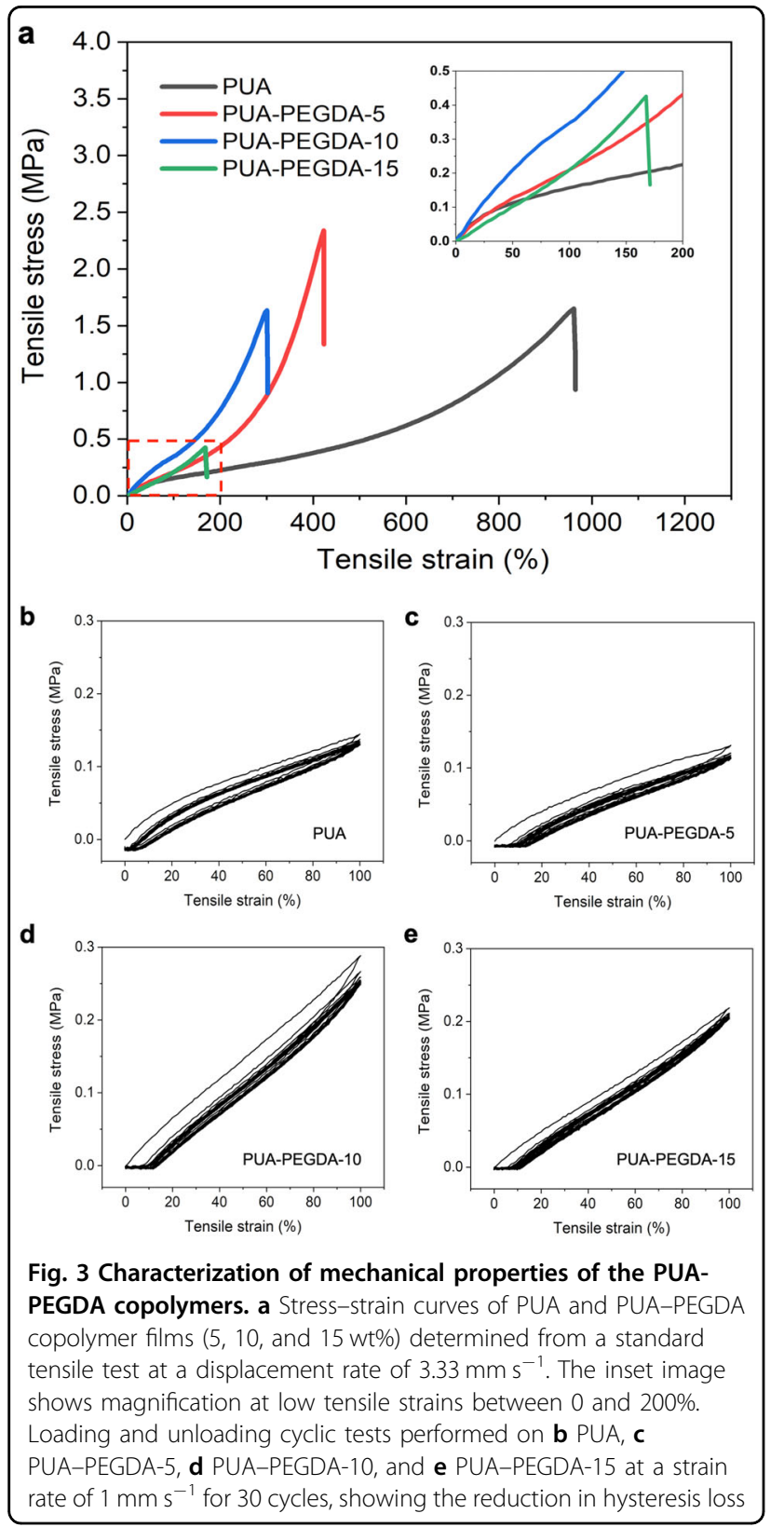

hysteresis loss, which is represented by the area enclosed in the curve. This area represents the energy dissipated during cycling, which is related to the internal friction and sliding of polymer chains in viscoelastic elastomers. As a result of the Mullins effect, a large hysteresis loss during the first cycle was observed for all samples, wherein the largest loss was displayed by the pristine PUA sample. After the second cycle, the stress-softening behavior during cyclic loading begins to reach a stationary state with a stable hysteresis loop being formed ${ }^{24}$. A reduction in hysteresis loss after the first cycle can be observed with increasing PEGDA concentration, which occurs because the increasing crosslinking density between polymer chains restricts motion and lowers the dissipative energy. Moreover, the reduction in hysteresis implies a reduced viscoelastic loss, which can be correlated to improved electromechanical stability through cycling tests of up to 1050 cycles (Figs. S7-10, Supplementary Information); this phenomenon aided in the enhanced dynamic response times of the actuator, which will be elaborated later.

\section{Static performance of buckling-mode DEA}

To demonstrate an entirely flexible device, a bucklingmode actuator was set up under prestretch-free conditions. Carbon grease was coated on both surfaces with a circular overlapping region, which is defined as the electroactive region. Buckling of the film occurs as the electroactive region expands against the passive region of the DEA with no overlapping electrodes ${ }^{25}$. This phenomenon creates a boundary condition that suppresses the lateral expansion of the electroactive region and generates a compressive stress across the DE. When the buckling limit is exceeded, out-of-plane deflection is achieved as the film buckles ${ }^{26}$. The actuated out-of-plane displacement was measured using a laser displacement sensor (Fig. 4a, b). Area strains were determined from changes in the electroactive areas through geometric relations, whereas deflection strains (Fig. S5) were based on deflection-to-thickness ratios. Figure 4c and Fig. S6 show that the pristine PUA exhibits a sudden increase in area strain before dielectric breakdown, which was not

Table 1 Mechanical properties of PUA and PUA-PEGDA copolymers

\begin{tabular}{llllll}
\hline Materials & Break tensile strength [MPa] & Elongation at break [\%] & $\mathbf{Y}_{\mathbf{1 0 \%}}{ }^{\mathbf{a}}[\mathbf{M P a}]$ & $\mathbf{Y}_{\mathbf{5 0 \%}}{ }^{\mathbf{b}}$ [MPa] & Hysteresis loss [\%] \\
\hline PUA & 1.65 & 960 & 0.337 & 0.100 & 18.74 \\
PUA-PEGDA-5 & 2.33 & 423 & 0.337 & 0.148 & 14.25 \\
PUA-PEGDA-10 & 1.64 & 301 & 0.550 & 0.330 & 8.40 \\
PUA-PEGDA-15 & 0.42 & 168 & 0.323 & 0.168 & 6.91 \\
\hline
\end{tabular}

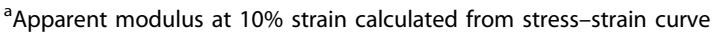

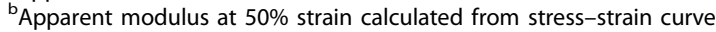




\section{a}

b

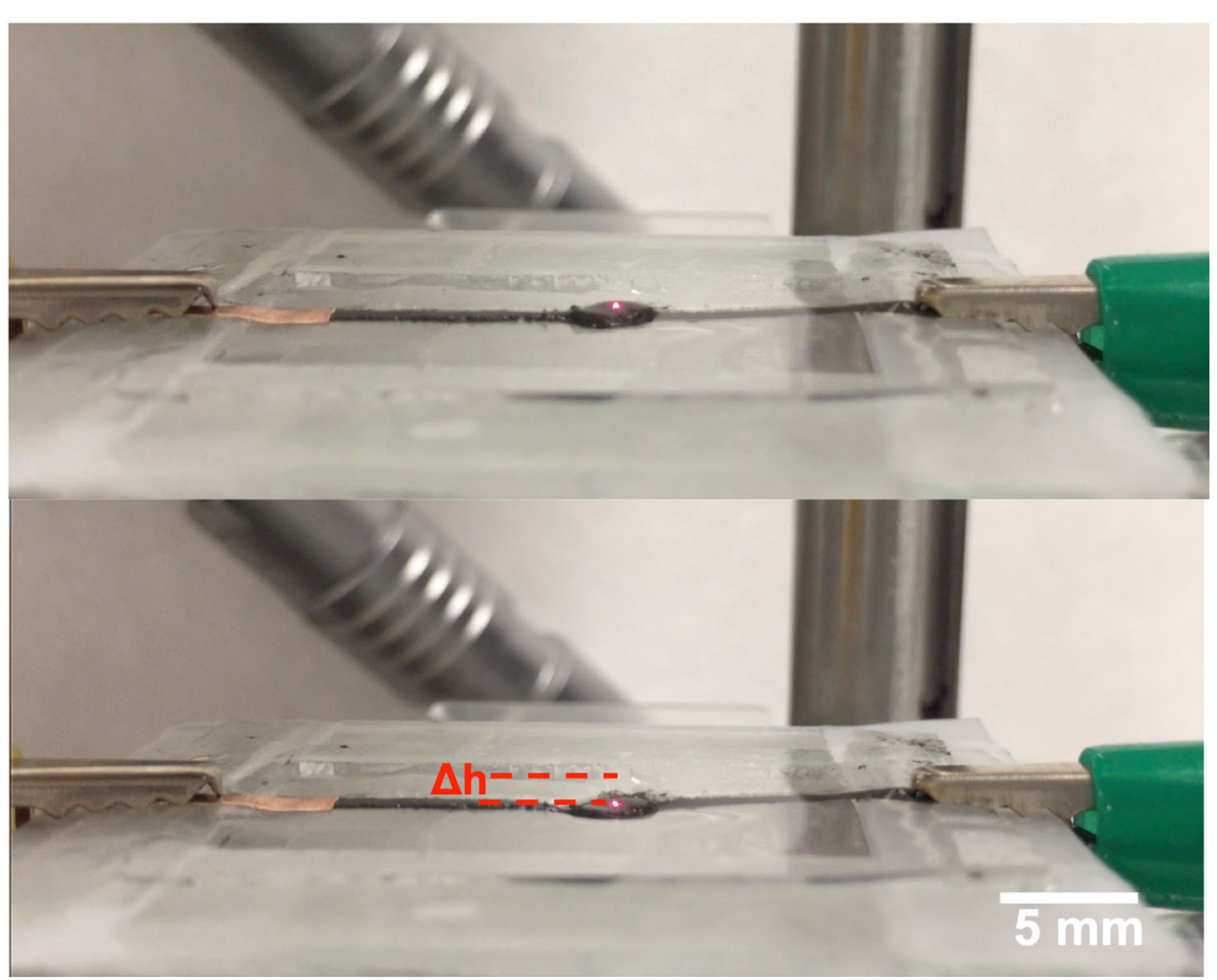

c

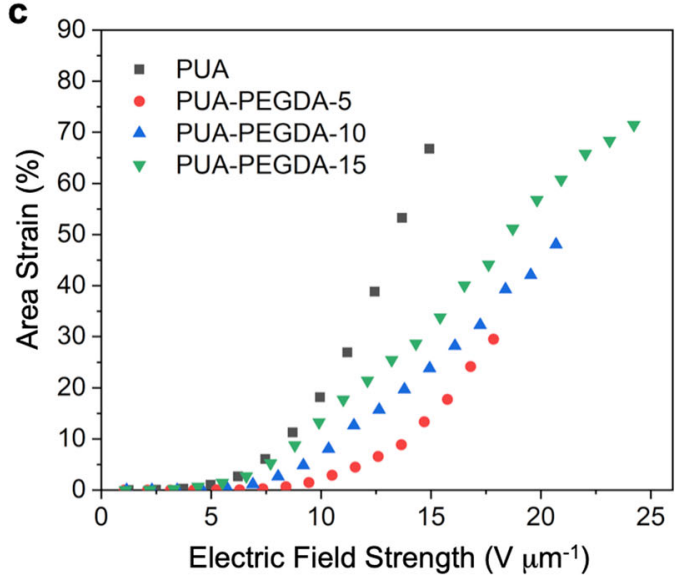

d

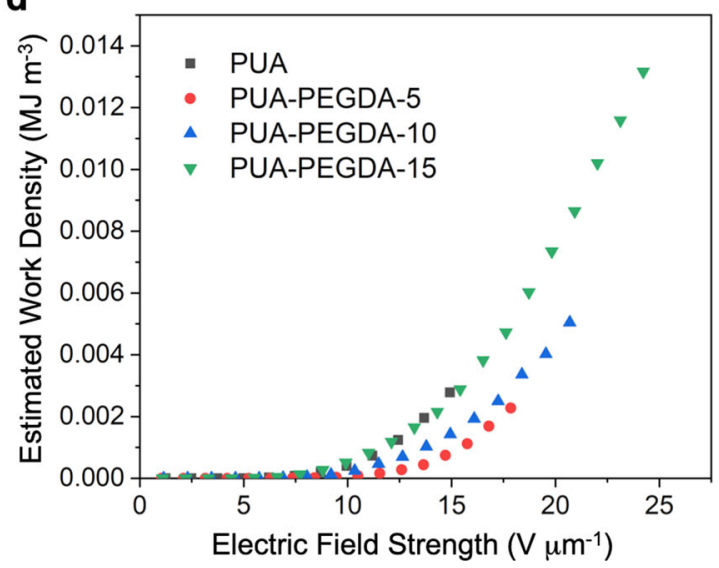

Fig. 4 Static actuation performance of the buckling-mode DEAs. Actuation of a single buckling-mode dielectric elastomer actuator (DEA) in the $\mathbf{a}$ off state and $\mathbf{b}$ on state, where $\triangle h$ represents the displacement achieved when a voltage is applied. $\mathbf{c}$ Representative area strains of PUA and PUA-PEGDA copolymers (5, 10, and 15 wt\%) with increasing electric field strength. $\mathbf{d}$ Estimated work density based on measured area strains of PUA and PUA-PEGDA copolymers $(5,10$, and $15 \mathrm{wt} \%)$ with increasing electric field strength

observed in the PUA-PEGDA copolymers (PUA-PEGDA-X, where $\mathrm{X}$ is the wt\% of the PEGDA). This finding is attributed to the nonlinear behavior of PUA, for which the modulus becomes significantly smaller than that of the PUA-PEDGA copolymers (Fig. 3a) beyond $10 \%$ strain. The early breakdown of PUA is expected without the application of prestrains to prevent electromechanical instabilities. In contrast, copolymers with higher concentrations of PEGDA (10 wt\% and $15 \mathrm{wt}$ $\%$ ) could be tested beyond the breakdown strength of pristine PUA and PUA-PEGDA- 5 because the increase in stiffness from chemical crosslinks lowers the likelihood of electromechanical instabilities ${ }^{27}$. With this effect, PUA-PEGDA-10 and PUA-PEGDA-15 could be tested at higher electric field strengths to achieve higher maximum strains than PUA or PUA-PEGDA-5. A 
Table 2 Comparison of area strain between DEA materials without prestrains

\begin{tabular}{lllll}
\hline Materials & $\begin{array}{l}\text { Area } \\
\text { strain [\%] }\end{array}$ & $\begin{array}{l}\text { Electric } \\
\text { field } \\
{\left[\mathbf{V} \mathbf{~ m}^{-\mathbf{1}}\right]}\end{array}$ & $\begin{array}{l}\text { Dielectric } \\
\text { constant } \\
\text { (at } \mathbf{~} \mathbf{~ k H z} \text { ) }\end{array}$ & Reference \\
\hline PUA & 66.7 & 14.9 & 5.5 & This work \\
PUA-PEGDA-15 & 71.4 & 24.2 & 9.4 & This work \\
CCTO@PANI/ & 13.24 & 10 & $\sim 4.25$ & 28 \\
PDMS & & & & \\
AZO-g-PDMS & 17 & 68 & 4.64 & 16 \\
SiR-DN 2/1 & 11.2 & 15 & $\sim 10$ & 29 \\
C-P(BA-GMA)-3 & 52.1 & 21.6 & 5.67 & 30 \\
VHB 4910 & 7.5 & 17 & 4.2 & 31 \\
TPU 80 & 2.3 & 42 & 5.1 & 32 \\
PUU-HS-13 & $\sim 18$ & 40 & 7.2 & 33 \\
\hline
\end{tabular}

comparison between the area strains of the DEA materials with no prestrains is presented in Table 2. In comparison to low viscoelastic materials, such as silicone, PUA-PEGDA-15 displayed higher area strains under lower electric field strengths without prestretching. This finding can be attributed to the high dielectric permittivity, which led to a higher generated Maxwell pressure.

Under an isochoric assumption, the work density $\left(u_{\mathrm{e}}\right)$ of the material was calculated as the work done by the actuator divided by its volume, as shown in Eq. 3, where $P$ represents the generated Maxwell pressure and $s_{z}$ is the thickness strain determined from the measured area strains. $^{34}$

$$
u_{e}=-\frac{1}{2} P \ln \left(1+s_{z}\right)
$$

As shown in Fig. 4d, the reduction in the work density that arose from the increased crosslinking with increasing PEGDA concentration was gradually minimized. At electric field strengths of up to $15 \mathrm{~V} / \mu \mathrm{m}$, the work density of PUA-PEGDA-15 was similar to that of the pristine PUA sample, indicating an optimum balance between the effects of the increased dielectric constant and the elastic modulus. Furthermore, as PUA-PEGDA-15 could be tested at higher electric field strengths, the maximum work density achieved by PUA-PEGDA-15 was the highest among the measured samples; this evidently shows the effectiveness of polar crosslinks for increasing the load that the actuators are capable of driving.

\section{Dynamic performance of a buckling-mode DEA}

The dynamic performance and endurance of the buckling-mode actuator were evaluated through cyclic tests performed at a frequency of $0.5 \mathrm{~Hz}$ for 1050 cycles (Movie S1, Supplementary Information). To evaluate the effects of the viscoelastic drift, we presented the first 145 cycles (Fig. 5a-d); subsequent cycles are presented in Figs. S6-9 in the Supporting Information. Samples of PUA and PUA-PEGDA-5 were observed to gradually drift from their equilibrium position by a large amount $(>0.15 \mathrm{~mm})$ before reaching a stable state. Such behavior is the result of the inherent viscoelastic characteristics of the film, wherein the relaxation time is slower than the cycling time, and the energy is dissipated from the sliding of the polymer chains across each other. Similar behaviors have been observed in elastomers with high viscoelasticity, such as VHB polymers ${ }^{17}$. The introduction of PEGDA as chemical crosslinks, which anchor the polymer chains to reduce molecular motion, results in better thermal stability (Fig. S4), faster relaxation time and minimal drift of the DEA. These findings are evident from Fig. 5d, as the viscoelastic drift decreases significantly for the PUA-PEGDA-15 samples. At the end of 145 cycles, PUA drifted a distance of $0.28 \mathrm{~mm}$ from its initial start point, whereas PUA-PEGDA-15 drifted a distance of only $0.09 \mathrm{~mm}$. With reduced viscoelastic drift, better location precision can be obtained. In addition, by tuning the viscoelastic nature of the polymer films, the response times of the actuator can be controlled. To allow sufficient time for the peak displacement to be reached, the voltage was applied at $0.1 \mathrm{~Hz}$. The response time $t_{0.9}$ of the actuator was quantified as the time taken to reach $90 \%$ of the maximum displacement for each cycle. From Fig. 5f, PUA-PEGDA-15 achieved a response time of $<1 \mathrm{~s}$, which is an improvement compared to PUA; this finding was corroborated by the reduced hysteresis loss tested earlier. The strain response (Fig. 5e) of the synthesized PUA-PEGDA-15 is analogous to that of silicones with higher elastic properties, lower dissipative energy and a faster response time. ${ }^{3,35}$

To demonstrate the provision of pixelated actuation, we extended the localized actuator into an array through a cross-junction design configuration where three actuators can be activated simultaneously (Fig. 6a). The fabricated actuator array with a cross-junction configuration can be realized on a flexible substrate without a rigid frame or prestretching, as shown in Fig. $6 \mathrm{~b}$ and Movie S2 in the Supporting Information. The conceptualization of the fabricated actuator array integrated into a watch strap to provide haptic feedback for wearables can be found in Fig. 6c and Movie S3.

\section{Conclusion}

In this work, we presented an approach to tune the viscoelastic properties of a DEA for improved dynamic performance. Through the addition of low molecular weight PEGDA, we increased the number of chemical 

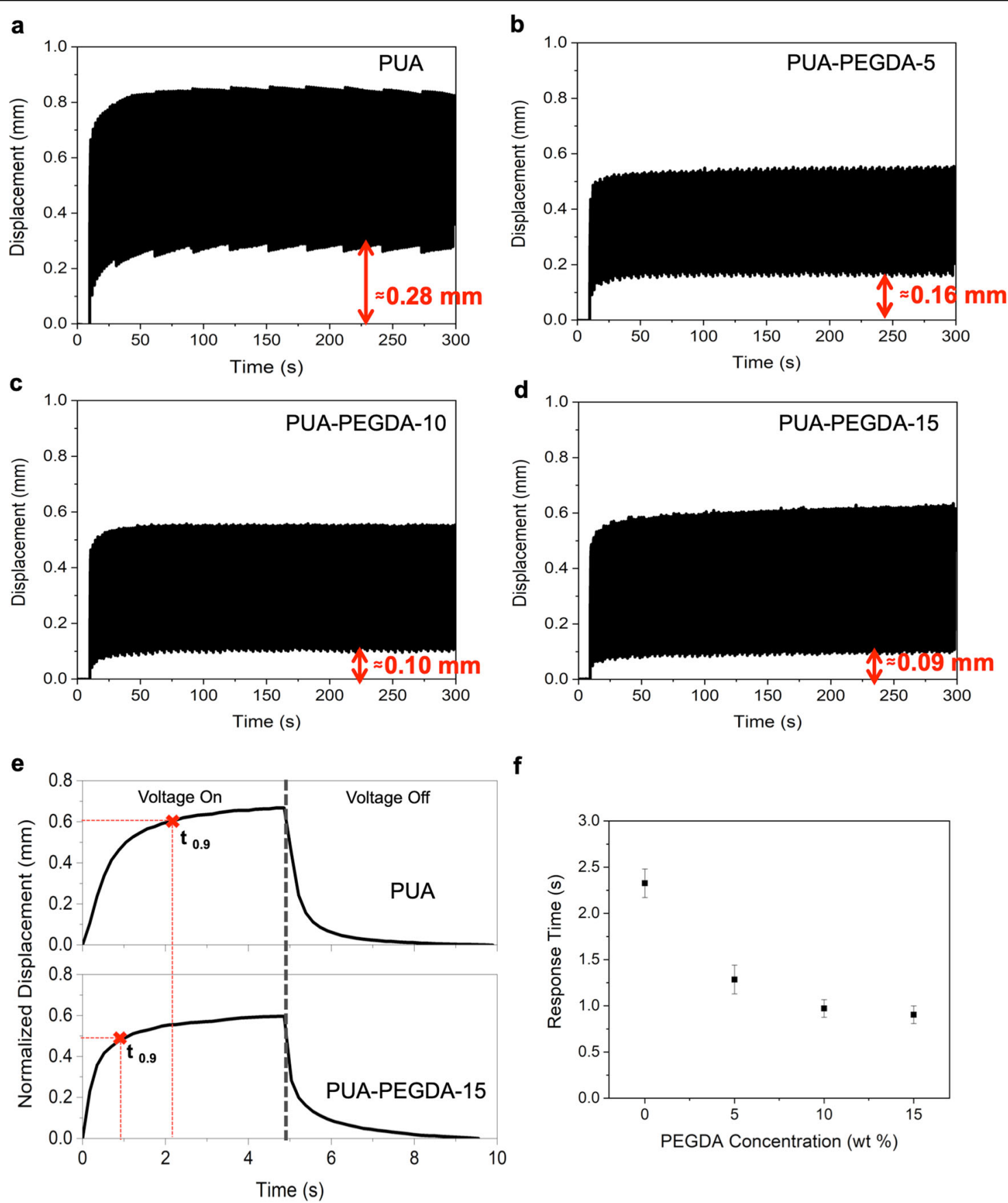

$\mathbf{f}$

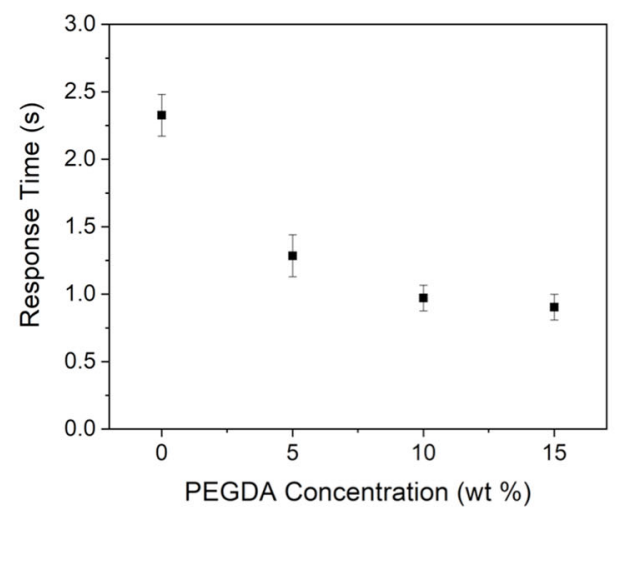

Fig. 5 Dynamic actuation performance of the buckling-mode DEAs. First, 145 cycles of the cyclic test were performed at an electric field strength of $10 \mathrm{~V} \mathrm{um}^{-1}$ at a frequency of $0.5 \mathrm{~Hz}$ for a PUA, b PUA-PEGDA-5, c PUA-PEGDA-10, and $\mathbf{d}$ PUA-PEGDA-15. The approximate drift distance after 145 cycles is stated in red. Profile of a single cycle of $\mathbf{e}$ the strain response of PUA and PUA-PEGDA-15 under a $10 \mathrm{~V} \mathrm{~mm}^{-1}$ electric field at $0.1 \mathrm{~Hz}$ is shown to demonstrate the difference in response time, $t_{0.9}$. This response time $t_{0.9}$ represents the time for the actuator to reach $90 \%$ of its maximum displacement at $0.1 \mathrm{~Hz}$. f Response time $t_{0.9}$ at various PEGDA concentrations $(0,5,10$, and $15 \mathrm{wt} \%)$

crosslinks within the polymer matrix, thereby anchoring the polymer chains. As a result, the slipping of the polymer chains across each other is minimized, thereby reducing the viscoelastic effects. This phenomenon is supported by the reduction in hysteresis loss during mechanical cyclic loading with increasing PEGDA concentrations as energy dissipation from molecular motion is reduced. To verify our hypothesis, we fabricated a buckling-mode actuator and performed cycling experiments. The response times were reduced by more than 

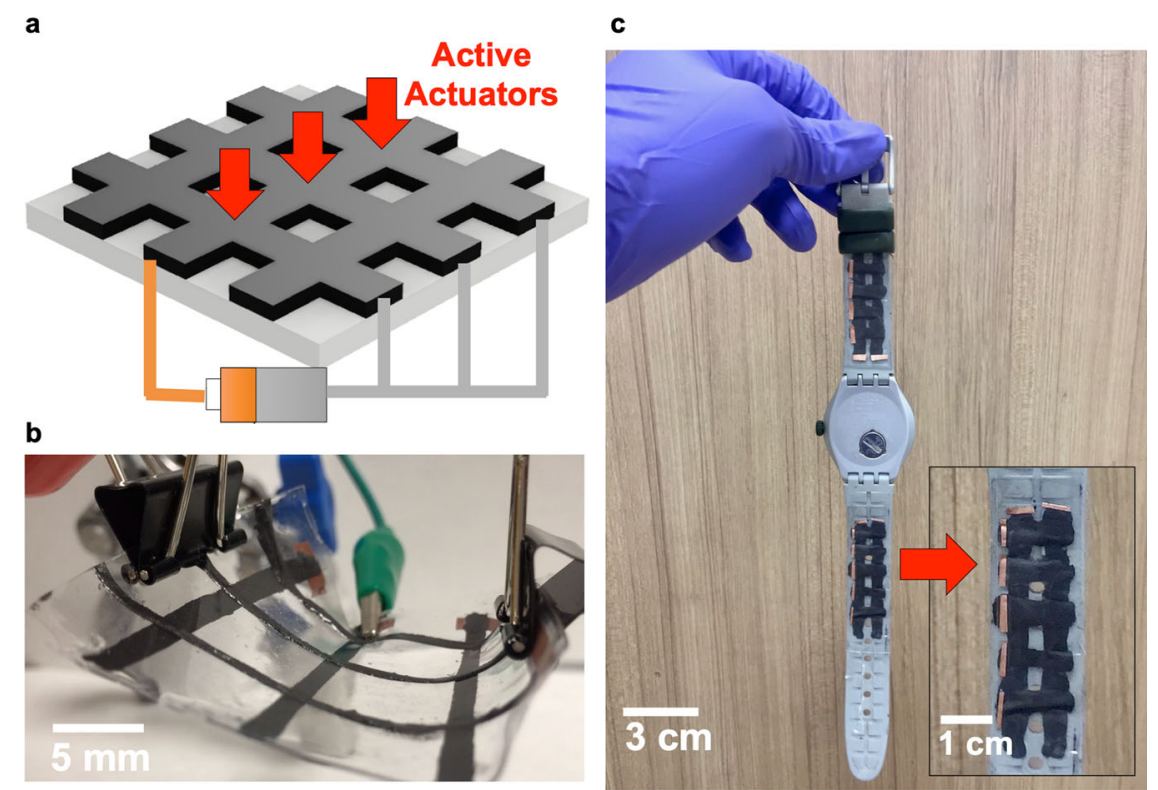

Fig. 6 a Schematic of an actuator array in a cross-junction configuration. Electrical connections are connected to activate the middle three actuators. b Fabricated actuator array in a cross-junction configuration on a flexible substrate. c Conceptualization of the fabricated actuator array integrated into a watch strap to provide haptic feedback for wearables

$50 \%$ with increasing PEGDA concentrations, reaching $90 \%$ of the maximum amplitude in $<1 \mathrm{~s}$. In addition, stable and reliable performance was observed with a significant reduction in viscoelastic drifts with increasing PEGDA concentration, allowing the potential usage of the proposed DEA for high precision applications, such as tunable lenses and gratings. Although the increase in the number of crosslinks was shown to increase the elastic modulus of the material, by introducing polar groups present in PEGDA, we were able to achieve a maximum area strain that was notably higher than low viscoelastic materials, such as silicone, which requires a larger operating voltage. Furthermore, our device avoids the use of rigid components used to achieve a prestretched state for reduced viscoelastic effects and improved actuation performances. This capability allows the fabricated copolymer to be integrated into soft and flexible bodies, highlighting a promising approach for faster, stable, and flexible actuators.

\section{Acknowledgements}

This research was financially supported by the Competitive Research Programme Award no. NRF-CRP-13-2014-02 and the NRF-Investigatorship Award no. NRF-NRFI2016-05 provided by the National Research Foundation, Singapore. M.W.M.T acknowledges the scholarship awarded by the Nanyang Technological University, Singapore.

Conflict of interest

The authors declare that they have no conflict of interest.

\section{Publisher's note}

Springer Nature remains neutral with regard to jurisdictional claims in published maps and institutional affiliations.

Supplementary information is available for this paper at https://doi.org/ 10.1038/s41427-019-0147-5.

Received: 18 February 2019 Revised: 20 May 2019 Accepted: 4 June 2019. Published online: 25 October 2019

\section{References}

1. Pelrine, R., Kornbluh, R. \& Kofod, G. High-strain actuator materials based on dielectric elastomers. Adv. Mater. 12, 1223-1225 (2000).

2. Pelrine, R. et al. High-field deformation of elastomeric dielectrics for actuators. Mater. Sci. Eng. C. 11, 89-100 (2000).

3. Biggs, J. et al. Electroactive polymers: developments of and perspectives for dielectric elastomers. Angew. Chem. Int. Ed. 52, 9409-9421 (2013).

4. Pelrine, R., Kornbluh, R., Pei, Q. \& Joseph, J. High-speed electrically actuated elastomers with strain greater than 100\%. Science 287, 836-839 (2000).

5. Kofod, G., Sommer-Larsen, P., Kornbluh, R. \& Pelrine, R. Actuation response of polyacrylate dielectric elastomers. J. Intell. Mater. Syst. Struct. 14, 787-793 (2003).

6. Romasanta, L. J., López-Manchado, M. A. \& Verdejo, R. Increasing the performance of dielectric elastomer actuators: a review from the materials perspective. Prog. Polym. Sci. 51, 188-211 (2015).

7. Zhang, X., Wissler, M., Jaehne, B., Breonnimann, R. \& Kovacs, G. Effects of crosslinking, prestrain, and dielectric filler on the electromechanical response of a new silicone and comparison with acrylic elastomer. Proc. SPIE 5385, 78-86 (2004).

8. Zhao, X., Koh, S. J. A. \& Suo, Z. Nonequilibrium thermodynamics of dielectric elastomers. Int. J. Appl. Mech. 3, 203-217 (2011).

9. Foo, C. C., Cai, S., Koh, S. J. A., Bauer, S. \& Suo, Z. Model of dissipative dielectric elastomers. J. Appl. Phys. 111, 034102 (2012).

10. Zou, J. \& Gu, G. Modeling the viscoelastic hysteresis of dielectric elastomer actuators with a modified rate-dependent Prandtl-Ishlinskii model. Polymers 10, 525 (2018). 
11. Huang, X., Xie, L., Hu, Z. \& Jiang, P. Influence of $\mathrm{BaTiO}_{3}$ nanoparticles on dielectric, thermophysical and mechanical properties of ethylene-vinyl acetate elastomer/BaTiO3 microcomposites. IEEE Trans. Dielectr. Electr. Insul. 18 375-383 (2011).

12. Yang, D. et al. Improved electromechanical properties of silicone dielectric elastomer composites by tuning molecular flexibility. Compos. Sci. Technol. 155, 160-168 (2018)

13. Hu, W., Zhang, S. N., Niu, X., Liu, C. \& Pei, Q. An aluminum nanoparticle-acrylate copolymer nanocomposite as a dielectric elastomer with a high dielectric constant. J. Mater. Chem. C. 2, 1658-1666 (2014).

14. Poikelispää, M., Shakun, A., Das, A. \& Vuorinen, J. Improvement of actuation performance of dielectric elastomers by barium titanate and carbon black fillers. J. Appl. Polym. Sci. 133, 44116 (2016).

15. Kim, J. Y., Park, S. H. \& Yu, S. Effect of chlorine-containing polymer additive on dielectric performance of polymer dielectric films. Electron. Lett. 50, 357-358 (2014).

16. Zhang, L. et al. Highly improved electro-actuation of dielectric elastomers by molecular grafting of azobenzenes to silicon rubber. J. Mater. Chem. C. 3 4883-4889 (2015).

17. Liu, L. et al. Experimental study on the dynamic response of in-plane deformation of dielectric elastomer under alternating electric load. Smart Mater. Struct. 23, 025037 (2014)

18. Tran, D. Q., Li, J., Xuan, F. Z. \& Xiao, T. Viscoelastic effects on the actuation performance of a dielectric elastomer actuator under different equal, un-equal biaxial pre-stretches. Mater. Res. Express 5, 065303 (2018).

19. Jiang, L. et al. Electromechanical instability in silicone-and acrylate-based dielectric elastomers. J. Appl. Polym. Sci. 135, 45733 (2018).

20. Kanyanta, V. \& Ivankovic, A. Mechanical characterisation of polyurethane elastomer for biomedical applications. J. Mech. Behav. Biomed. Mater. 3, 51-62 (2010).

21. Sahu, R. K., Patra, K., Bhaumik, S., Pandey, A. K. \& Setua, D. K. Stress-strain behaviour of dielectric elastomer for actuators. Appl. Mech. Mater. 789, 837 (2015).

22. Johnston, I. D., McCluskey, D. K., Tan, C. K. L. \& Tracey, M. C. Mechanical characterization of bulk Sylgard 184 for microfluidics and microengineering. J. Micromech. Microeng. 24, 035017 (2014).
23. Martinez, R. V., Glavan, A. C., Keplinger, C., Oyetibo, A. I. \& Whitesides, G. M. Soft actuators and robots that are resistant to mechanical damage. Adv. Funct. Mater. 24, 3003-3010 (2014).

24. Diani, J., Fayolle, B. \& Gilormini, P. A review on the Mullins effect. Eur. Polym. J. 45, 601-612 (2009).

25. Bense, H., Trejo, M., Reyssat, E., Bico, J. \& Roman, B. Buckling of elastomer sheets under non-uniform electro-actuation. Soft Matter 13, 2876-2885 (2017).

26. Rosset, S., Niklaus, M., Dubois, P. \& Shea, H. R. Large-stroke dielectric elastomer actuators with ion-implanted electrodes. J. Micro. Syst. 18, 1300-1308 (2009).

27. Zhao, X. \& Suo, Z. Theory of dielectric elastomers capable of giant deformation of actuation. Phys. Rev. Lett. 104, 178302 (2010).

28. Zhang, Y. Y. et al. Preparation and properties of core-shell structured calcium copper titanate@polyaniline/silicone dielectric elastomer actuators. Polym. Compos. 40, E62-E68 (2019).

29. Sun, H. et al. Simultaneously improved dielectric and mechanical properties of silicone elastomer by designing a dual crosslinking network. Polym. Chem. 10, 633-645 (2019).

30. Zhao, Y., Zha, J. W., Yin, L. J., Gao, Z. S., Wen, Y. Q. \& Dang, Z. M. Remarkable electrically actuation performance in advanced acrylic-based dielectric elastomers without pre-strain at very low driving electric field. Polymer. 137, 269-275 (2018).

31. Shankar, R., Ghosh, T. K. \& Spontak, R. J. Electromechanical response of nanostructured polymer systems with no mechanical pre-strain. Macromol. Rapid Commun. 28, 1142-1147 (2007).

32. Renard, C. et al. Remarkably improved electromechanical actuation of polyurethane enabled by blending with silicone rubber. RSC Adv. 7, 22900-22908 (2017).

33. Xiang, D. et al. Optimization of mechanical and dielectric properties of poly (urethane-urea)-based dielectric elastomers via the control of microstructure RSC Adv. 7, 55610-55619 (2017).

34. Ha, S. M. et al. High electromechanical performance of electroelastomers based on interpenetrating polymer networks. Proc. SPIE 6927, 69272C (2008).

35. Michel, S., Zhang, X. Q., Wissler, M., Löwe, C. \& Kovacs, G. A comparison between silicone and acrylic elastomers as dielectric materials in electroactive polymer actuators. Polym. Int. 59, 391-399 (2010). 